\title{
Facteurs de risque et dépistage du cancer chez les membres des Premières Nations vivant hors réserve, chez les Métis et chez les non-Autochtones en Ontario
}

\author{
D. R. Withrow, M. Sc. (1, 2); A. Amartey, M.H.P. (1); L. D. Marrett, Ph. D. (1, 2)
}

Cet article a fait l'objet d'une évaluation par les pairs.

\section{Résumé}

Introduction : Cette étude vise à décrire la prévalence du tabagisme, de l'obésité, des comportements sédentaires et de l'activité physique, de la consommation de fruits et de légumes et de la consommation d'alcool ainsi que la participation au dépistage du cancer du sein, du cancer du col de l'utérus et du cancer colorectal chez les adultes des Premières Nations et métis en Ontario, par rapport à la population non autochtone.

Méthodologie : Nous avons utilisé les données de l’Enquête sur la santé dans les collectivités canadiennes (données de 2007 à 2011 combinées) pour calculer les estimations de la prévalence chez les trois populations ethnoculturelles.

Résultats : Par rapport aux adultes non autochtones, les adultes des Premières Nations et métis étaient beaucoup plus nombreux à déclarer fumer et à être classés comme obèses. Les membres des Premières Nations étaient plus nombreux que la population non autochtone à consommer de l'alcool au-delà des recommandations en matière de prévention du cancer et à consommer insuffisamment des fruits et des légumes. Les femmes des Premières Nations étaient plus nombreuses que les femmes non autochtones à déclarer avoir fait l'objet d'un dépistage par recherche de sang occulte dans les selles au cours des deux dernières années. Aucune différence significative n’a été observée entre les membres des trois groupes ethnoculturels en ce qui concerne le dépistage du cancer du sein et du cancer du col de l'utérus chez les femmes et le dépistage du cancer colorectal chez les hommes.

Conclusion : Si l'on intervient pas, il est probable que les cancers liés au tabagisme et à l'obésité continueront à représenter un fardeau considérable au sein de la population autochtone de l'Ontario.

Mots-clés : cancer, maladies chroniques, groupe d'ascendance autochtone de l'Amérique du Nord, facteurs de risque, dépistage de masse, population autochtone, Premières Nations, Métis, Ontario

\section{Introduction}

Le cancer est l'une des principales causes de décès chez les Autochtones ${ }^{1}$. Traditionnellement, l'incidence du cancer au Canada était moins élevée chez les Autochtones, mais elle augmente à un rythme plus rapide que chez la population non autochtone, pour laquelle les taux de cancer ont été relativement stables au cours des deux dernières décennies ${ }^{2,3}$. Il est important de connaître la prévalence des facteurs de risque du cancer et la participation au dépistage du cancer chez les sous-populations autochtones pour pouvoir étayer l'élaboration de stratégies de contrôle et de prévention du cancer destinées aux Autochtones.

Les enquêtes sur la santé de la population sont une source de données souvent utilisée pour évaluer la prévalence des facteurs de risque au sein de la population générale. Cependant, leur utilisation pour l'étude de la santé chez les Autochtones est problématique. Bien que plusieurs enquêtes réalisées au cours des dernières années aient inclus des variables ethnoculturelles pour identifier les répondants autochtones, les enquêtes nationales sur la santé de la population sont généralement menées auprès d'un échantillon relativement petit d'Autochtones. Comme la prestation des services de santé destinés aux Autochtones relève de plus en plus du champ de compétence provinciale ${ }^{4}$, il importe de disposer de statistiques provinciales en matière de santé des souspopulations. Cependant, la stratification par province des enquêtes populationnelles nationales a pour effet de limiter davantage le nombre de répondants autochtones disponibles. Par exemple, bien que l'Ontario compte la plus grande population d'Autochtones au Canada, soit environ 200000 membres des Premières Nations et 86000 Métis $^{5}$, la taille de l'échantillon de répondants autochtones interrogés dans le cadre d'une enquête nationale comme l’Enquête sur la santé dans les collectivités canadiennes (ESCC) demeure insuffisante. L'ESCC sonde environ 21000 répondants en Ontario chaque année, dont environ 600 se déclarent Autochtones, ce qui est

Rattachement des auteurs :

1. Surveillance et prévention, Prévention et lutte contre le cancer, Action Cancer Ontario, Toronto (Ontario), Canada 2. Département d'épidémiologie, École de santé publique Dalla Lana, Université de Toronto, Toronto (Ontario), Canada

Correspondance : Diana R. Withrow, Surveillance et prévention, Prévention et lutte contre le cancer, Action Cancer Ontario, 505, avenue University, Toronto (Ontario) M5G 1X3; tél. : 416-971-9800, poste 3125; téléc. : 416-971-6888; courriel : diana.withrow@cancercare.on.ca 
insuffisant pour fournir des estimations interprétables et significatives à propos des Premières Nations et des Métis de l'Ontario pour une année d'enquête donnée.

Pour remédier à ce problème de petite taille des échantillons, nous avons pris appui sur les travaux récents de Statistique Canada qui regroupent les données recueillies dans le cadre de plusieurs ESCC $^{6}$. Nous avons également utilisé d'autres données plus récentes (de 2011) en prêtant une attention particulière aux facteurs de risque associés au cancer, en estimant la participation aux divers tests de dépistage du cancer et en tenant compte de l'incidence des facteurs sociodémographiques. Nos travaux visaient plus particulièrement d'abord à mesurer la prévalence du tabagisme, de l'obésité, de l'activité physique, du sédentarisme, de la consommation de fruits et de légumes ainsi que de la consommation d'alcool chez les membres des Premières Nations et les Métis de l'Ontario, et à comparer ces facteurs de risque de cancer $^{7-12}$ à ceux de la population non autochtone, puis à mesurer les taux de dépistage les plus récents du cancer colorectal, du cancer du sein et du cancer du col de l'utérus chez les membres des Premières Nations et les Métis de l'Ontario et à les comparer à ceux de la population non-autochtone. Dans les deux cas, nous avons tenu compte de l'incidence des facteurs sociodémographiques. Grâce à cette analyse, nous espérons mettre en évidence les domaines d'intervention pour la lutte contre le cancer chez les Autochtones et fournir une base de référence aux mesures futures de ces éléments.

\section{Méthodologie}

\section{Sources de données}

Notre étude s'appuie sur les données recueillies par Statistique Canada entre 2007 et 2011 dans le cadre de l'ESCC. Cette dernière visait la population de 12 ans et plus vivant dans les 10 provinces et les territoires canadiens, à l'exception des personnes en établissement, de la population vivant dans les régions sanitaires du Nunavik et des Terres-Cries-de-la-BaieJames au Québec et de la population vivant dans les réserves ou d'autres lieux de peuplement autochtone des provinces. Les répondants de l'enquête ont été sélectionnés à partir de trois bases de sondage et $99 \%$ de l'échantillon provenait d'une base de sondage aréolaire ou d'une base de liste de numéros de téléphone. Environ $1 \%$ de l'échantillon provenait d'une base de sondage à composition aléatoire. Le questionnaire a été administré au moyen d'entrevues assistées par ordinateur et un nombre approximativement égal de répondants a été interrogé en personne et par téléphone ${ }^{13}$.

Pour cette étude, nous avons limité l'échantillon aux répondants adultes de la province de l'Ontario. Entre 2007 et 2011, le taux de réponse en Ontario a varié de $68,7 \%$ à 73,6 $\%{ }^{13}$. Pour augmenter le nombre de réponses admissibles à l'analyse, nous avons combiné les fichiers de microdonnées de l'ensemble des cinq questionnaires annuels de l'ESCC (2007 à 2011) et les estimations de la prévalence ont été calculées pour l'ensemble de ces 5 années, conformément à la méthode décrite par Statistique Canada ${ }^{14}$.

\section{Mesure des facteurs de risque et de la participation au dépistage}

Nous avons calculé la prévalence de six facteurs de risque et de trois méthodes de dépistage. Sauf indication contraire, les analyses des facteurs de risque visaient les répondants âgés de 18 ans et plus. Les répondants ayant omis de répondre ou ayant fourni une réponse invalide à une question ont été exclus du dénominateur de l'indicateur comportant cette question. Les limites d'âge et les seuils de réponse pour chaque mesure de dépistage ont été basés sur les lignes directrices en vigueur en Ontario pour chaque méthode de dépistage $^{15-17}$. Les questions pertinentes à propos des comportements sédentaires et du dépistage du cancer du sein et du cancer du col de l'utérus ont été posées seulement dans les enquêtes de 2007, 2008 et 2011.

Les définitions de chaque indicateur sont décrites de façon plus détaillée ci-dessous.

- Nous avons défini l'indicateur de tabagisme comme la proportion de répondants âgés de 20 ans ou plus ayant déclaré fumer quotidiennement ou à l'occasion. Nous avons fixé l'âge limite à 20 ans par souci de cohérence avec d'autres indicateurs de la santé publique utilisés en Ontario ${ }^{18}$.

- Nous avons défini l'indicateur d'obésité comme la proportion de répondants qui, selon la taille et le poids déclarés, présentaient un indice de masse corporelle (IMC) de $30 \mathrm{~kg} / \mathrm{m}^{2}$ ou plus. Les femmes enceintes ou qui allaitent ont été exclues.

- Nous avons défini l'indicateur de comportement sédentaire comme la proportion de répondants qui passait au moins 11 heures par semaine devant un ordinateur ou au moins 15 heures par semaine à regarder la télévision en dehors de l'école ou du lieu de travail.

- Nous avons défini l'indicateur d'activité physique durant les loisirs comme la proportion de répondants classés dans les catégories modérément actifs ou actifs en ce qui concerne les loisirs au cours des 3 mois précédents, en fonction d'une estimation de la dépense énergétique (DE) quotidienne supérieure à $1,5 \mathrm{kcal} / \mathrm{kg} /$ jour. Pour déterminer la $\mathrm{DE}$, les répondants devaient indiquer le nombre de fois qu'ils avaient participé à différentes activités (notamment la natation, le patin à glace, le volleyball) et la durée moyenne de leur participation. La DE a été calculée en combinant cette information et l'équivalent métabolique de l'activité tenant compte de l'intensité de l'activité.

- Nous avons défini l'indicateur de consommation de fruits et légumes comme la proportion de répondants ayant consommé des fruits et légumes (sauf les pommes de terre) au moins cinq fois par jour en fonction d'une version abrégée d'un questionnaire sur la fréquence de consommation d'aliments. De la même manière que les documents de l'ESCC, dans lesquels les termes " fois » et « portions » sont utilisés de façon interchangeable, nous utilisons surtout le terme " portions » dans cet article. Cependant, il est important de noter que l'ESCC demande aux répondants combien de fois ils ont consommé un fruit ou légume donné au cours de la période visée par l'enquête.

- Nous avons défini l'indicateur de consommation quotidienne d'alcool comme la proportion de femmes ayant bu en moyenne plus d'une consomma- 
tion par jour et la proportion d'hommes ayant bu en moyenne plus de deux consommations par jour au cours de la semaine précédant l'entrevue. Ce seuil était fondé sur les recommandations en matière de prévention du cancer selon lesquelles il ne faut pas dépasser cette quantité $^{19}$. Les femmes enceintes ont été exclues.

- Nous avons défini l'indicateur de participation au dépistage du cancer du col de l'utérus comme la proportion de femmes âgées de 21 à 69 ans ayant déclaré avoir subi un test de Pap au cours des trois dernières années.

- Nous avons défini l'indicateur de participation au dépistage du cancer du sein comme la proportion de femmes âgées de 50 à 74 ans ayant déclaré avoir subi une mammographie au cours des deux dernières années. Pour ne tenir compte que des mammographies de dépistage et non celles d'enquêtes diagnostiques, nous avons exclu les femmes ayant déclaré avoir subi une mammographie pour une masse dépistée lors d'une consultation précédente, pour le suivi d'un traitement contre le cancer du sein, pour un problème mammaire ou pour une " autre raison [que le dépistage] ».

- Nous avons procédé au calcul de deux indicateurs pour le dépistage du cancer colorectal. D'abord, nous avons mesuré la proportion de répondants âgés de 50 à 74 ans ayant déclaré avoir subi un dépistage par recherche de sang occulte dans les selles (RSOS) au cours des deux dernières années. Nous avons ensuite calculé la proportion d'adultes dans ce groupe d'âge n'ayant pas subi un dépistage de ce type au cours des deux dernières années, n’ayant pas subi de coloscopie ou de sigmoïdoscopie au cours des 10 dernières années et pour qui il était temps de procéder à un test de dépistage du cancer colorectal.

\section{Identité autochtone}

Entre 2007 et 2010, tous les répondants aux ESCC devaient répondre à la question : « Êtes-vous un(e) Autochtone, c'est-à-dire, Première Nation (Indien(ne) de l'Amérique du Nord), Métis(se) ou Inuit(e)? ». Lorsqu'un participant répondait par l'affirmative, on lui demandait de préciser à quelle sous-population il appartenait. En 2011, cette question a été posée seulement aux répondants qui avaient indiqué précédemment être nés au Canada, aux États-Unis, en Allemagne ou au Groenland. Par souci d'uniformité, nous avons classé les répondants des enquêtes de 2007 à 2010 comme étant membres des Premières Nations ou des Métis uniquement s'ils avaient indiqué être nés dans l'un de ces quatre pays ${ }^{20}$.

Nous avons utilisé des catégories ethnoculturelles mutuellement exclusives malgré le fait que les répondants aient pu déclarer plusieurs identités autochtones. Par exemple, un répondant ayant déclaré être membre des Premières Nations ou des Premières nations et des Inuits a été classé comme membre des Premières Nations. Un répondant ayant indiqué être Métis seulement ou Métis et toute autre identité autochtone a été classé comme Métis. En raison de la petite taille des échantillons, nous n'avons pas généré d'estimation pour la catégorie Inuits pour cet article. Nos définitions des Premières Nations et des Métis sont illustrées de façon plus détaillée dans le tableau 1.

\section{Analyse statistique}

L’ESCC présente un plan d'échantillonnage complexe à plusieurs degrés. Les coeffi- cients de pondération (ou poids) attribués par Statistique Canada sont utilisés pour rendre compte de la probabilité de sélection, de la non-réponse et de la non-couverture. Dans cette étude, des poids ont également été ajustés pour prendre en compte la fusion de plusieurs cycles de l'enquête ${ }^{14}$. Nous avons employé les méthodes bootstrap pour calculer le coefficient de variation (CV) et les intervalles de confiance (IC) à $95 \%$. Conformément aux règlements de Statistique Canada, les estimations ayant un CV compris entre $16,6 \%$ et $33,3 \%$ sont identifiées et doivent être utilisées avec prudence. Celles ayant un CV supérieur à 33,3\% ont été supprimées en raison de l'extrême variabilité de l'échantillonnage.

Les estimations ont été standardisées selon l'âge, conformément à la structure démographique de la population autochtone de l'Ontario et aux données du recensement de 2006, en utilisant les groupes d'âge suivants : 20 à 24 ans, 25 à 44 ans, 45 à 64 ans et 65 ans et plus. Pour les indicateurs dont les seuils d'âge ne concordaient pas avec ceux utilisés dans le recensement, nous avons classé les répondants dans le groupe d'âge le plus proche pour cette standardisation selon l'âge (p. ex., les répondants âgés de 18 et 19 ans ont été classés comme faisant partie du groupe des 20 à 24 ans).
TABLEAU 1

Définition de l'identité des membres des Premières Nations (hors réserve), des Métis et des non-Autochtones en fonction des réponses à l'Enquête sur la santé dans les collectivités canadiennes

\begin{tabular}{|c|c|c|c|c|c|}
\hline & \multirow{2}{*}{$\begin{array}{c}\text { Identité } \\
\text { autochtone }\end{array}$} & \multicolumn{4}{|c|}{ Identité liée à un sous-groupe ${ }^{b}$} \\
\hline & & Unique & & Itiple & \\
\hline \multirow{2}{*}{$\begin{array}{l}\text { Premières Nations } \\
\text { (hors réserve) }\end{array}$} & Oui & Premières Nations & & & \\
\hline & Oui & & Premières Nations & & Inuit \\
\hline \multirow{4}{*}{ Métis } & Oui & Métis & & & \\
\hline & Oui & & Premières Nations & Métis & \\
\hline & Oui & & & Métis & Inuit \\
\hline & Oui & & Premières Nations & Métis & Inuit \\
\hline & Non & \multicolumn{4}{|c|}{ Né dans tout pays } \\
\hline Non-Autochtone & Oui & \multicolumn{4}{|c|}{$\begin{array}{l}\text { Né à l'extérieur du Canada, des États-Unis, de l'Allemagne et du } \\
\text { Groenland }\end{array}$} \\
\hline
\end{tabular}

a Les répondants devaient répondre à la question suivante : « Êtes-vous un Autochtone, c'est-à-dire un Indien de l'Amérique du Nord, un Métis ou un Inuit?».

b Les répondants devaient répondre à la question suivante : "Êtes-vous Indien(ne) de l'Amérique du Nord (Premières Nations)? », « Êtes-vous Métis(se)? », " Êtes-vous Inuit(e) (Inuk)? » et pouvaient fournir plusieurs réponses affirmatives. Les cases gris foncé sont utilisées parce que les réponses concernant l'identité unique et l'identité multiple sont mutuellement exclusives. 
Nous avons utilisé une régression logistique pour calculer les rapports de cotes (RC) exprimant la prévalence des facteurs de risque et la participation au dépistage chez les Premières Nations et les Métis par rapport à la population non autochtone de l'Ontario. Nous présentons les RC normalisés en fonction de l'âge selon le sexe et les RC normalisés en fonction de l'âge, du quintile de revenu, du niveau de scolarité et du lieu de résidence (milieu rural ou urbain) conformément à l'ESCC. L'année de l'ESCC a fait l'objet d'un contrôle dans les deux modèles. Les estimations ont été considérées comme significativement différentes sur le plan statistique par rapport aux données de référence lorsqu'aucun chevauchement n'a été observé entre les IC à $95 \%$ ou les RC $(1,00)$.

\section{Résultats}

En combinant cinq années de données de l'ESCC en Ontario, nous avons obtenu pour l'analyse 90866 répondants âgés de 18 ans et plus, dont 1468 s'étaient identifiés comme membres des Premières Nations vivant hors réserve et 990 comme Métis. Les caractéristiques démographiques des répondants appartenant à chaque groupe ethnoculturel sont résumées dans le tableau 2. Les populations des Premières Nations et des Métis étaient plus jeunes que les populations non autochtones, avaient un niveau de scolarité moins élevé et un revenu moindre et étaient plus susceptibles de vivre en milieu rural qu'urbain.

\section{Facteurs de risque}

Le tableau 3 ainsi que les figures 1 et 2 fournissent les estimations de prévalence des facteurs de risque et de participation au dépistage, tandis que le tableau 4 fournit les rapports de cotes. Les différences les plus marquées ont concerné la prévalence des facteurs de risque liés au tabagisme et à l'obésité entre les membres des Premières Nations, les Métis et les nonAutochtones. Chez les hommes, les membres des Premières Nations vivant hors réserve et les Métis étaient deux fois plus nombreux que leurs pairs non autochtones à déclarer fumer (RC Premières Nations = 2,33, IC à $95 \%: 1,79$ à 3,02; RC Métis = 2,09 , IC à $95 \%: 1,54$ à 2,83), avec 44,9\% (IC à $95 \%$ : 39,1 à 50,7) de membres des
Premières Nations et 42,9 \% (IC à $95 \%$ : 36,1 à 49,6) de Métis ayant déclaré fumer comparativement à 26,2 \% (IC à $95 \%$ : 25,3 à 27,1) des hommes non autochtones. Malgré une prévalence moins élevée du tabagisme chez les femmes, les disparités entre les groupes ethniques étaient supérieures à celles relevées chez les hommes. Les femmes des Premières Nations étaient environ 3,5 fois plus nombreuses ( $\mathrm{RC}=$ 3,56 , IC à $95 \%: 2,75$ à 4,61) que les femmes non autochtones à fumer, et les femmes métis étaient environ 2,5 fois plus nombreuses $(\mathrm{RC}=2,47$, IC à $95 \%: 1,86$ à 3,28 ) que les femmes non autochtones à fumer. Les membres des Premières Nations et les Métis étaient deux fois plus nombreux que les non-Autochtones à être considérés comme obèses. Les taux d'obésité variaient entre 16,0 \% chez les femmes non autochtones et $33,4 \%$ chez les hommes des Premières Nations.

Les hommes et les femmes des Premières Nations étaient considérablement moins nombreux que les non-Autochtones à

\section{TABLEAU 2}

Caractéristiques sociodémographiques des répondants à l'ESCC résidant en Ontario et âgés de 18 ans ou plus, selon le groupe d'identité autochtone (population hors réserve), données des ESCC de 2007 à 2011 combinées

\begin{tabular}{|c|c|c|c|c|c|}
\hline \multirow[t]{2}{*}{ Caractéristiques } & \multirow[t]{2}{*}{$\begin{array}{c}\text { Non- } \\
\text { Autochtones } \\
(n=88408)\end{array}$} & \multicolumn{2}{|c|}{$\begin{array}{l}\text { Premières Nations } \\
\text { (hors réserve) } \\
\text { (n=1468) }\end{array}$} & \multicolumn{2}{|c|}{$\begin{array}{c}\text { Métis } \\
(\mathbf{n}=990)\end{array}$} \\
\hline & & $\%$ & Valeur $p^{a}$ & $\%$ & Valeur $\boldsymbol{p}^{\mathrm{a}}$ \\
\hline \multicolumn{6}{|l|}{ Sexe } \\
\hline Masculin & 48,8 & 48,1 & & 46,0 & \\
\hline Féminin & 51,2 & 51,9 & 0,720 & 54,0 & 0,295 \\
\hline \multicolumn{6}{|l|}{ Groupe d'âge (ans) } \\
\hline 18 à 24 & 12,3 & 16,6 & & 10,5 & \\
\hline 25 à 44 & 36,0 & 41,5 & & 46,5 & \\
\hline 45 à 64 & 35,0 & 34,5 & & 35,1 & \\
\hline 65 et plus & 16,7 & 7,4 & $<0,001$ & 8,0 & $<0,001$ \\
\hline \multicolumn{6}{|l|}{ Niveau de scolarité } \\
\hline Sans diplôme d'études secondaires & 12,8 & 22,3 & & 21,6 & \\
\hline $\begin{array}{l}\text { Diplôme d'études secondaires/études } \\
\text { postsecondaires partielles }\end{array}$ & 26,3 & 30,2 & & 28,8 & \\
\hline Diplôme d'études postsecondaires & 61,0 & 47,5 & $<0,001$ & 49,6 & $<0,001$ \\
\hline \multicolumn{6}{|l|}{ Quintiles de revenu } \\
\hline 1 (le plus faible) & 18,3 & 33,1 & & 19,3 & \\
\hline 2 & 19,7 & 18,6 & & 24,1 & \\
\hline 3 & 20,3 & 17,0 & & 20,9 & \\
\hline 4 & 20,5 & 18,0 & & 18,8 & \\
\hline 5 (le plus élevé) & 21,3 & 13,3 & $<0,001$ & 16,9 & 0,097 \\
\hline \multicolumn{6}{|l|}{ Lieu de résidence $^{b}$} \\
\hline Urbain & 89,5 & 81,1 & & 78,4 & \\
\hline Rural & 10,5 & 18,9 & $<0,001$ & 21,6 & $<0,001$ \\
\hline
\end{tabular}

Source : Enquête sur la santé dans les collectivités canadiennes (données de 2007 à 2011 combinées), Statistique Canada.

Abréviations : AR, agglomération de recensement; ESCC, Enquête sur la santé dans les collectivités canadiennes; RMR, région métropolitaine de recensement.

a Les valeurs $p$ ont été générées au moyen d'un test du chi-carré $\left(\chi^{2}\right)$ pour calculer la différence de proportions entre les populations, c'est-à-dire entre les Premières Nations et les non-Autochtones, ainsi qu'entre les Métis et les non-Autochtones, respectivement.

b Le lieu de résidence urbain comprend les répondants vivant dans une RMR, une AR subdivisée en secteurs de recensement ou une AR non subdivisée en secteurs de recensement. Le lieu de résidence rural comprend les secteurs à l'extérieur d'une RMR et à l'extérieur d'une AR. 
TABLEAU 3

Prévalence, standardisée selon l'âge, des facteurs de risque sélectionnés et de la participation au dépistage du cancer chez la population adulte (âgée de 18 ans ou plus, à moins d'indication contraire) de l'Ontario vivant hors réserve, selon le groupe d'identité autochtone, données des ESCC de 2007 à 2011 combinées

\begin{tabular}{|c|c|c|c|c|c|c|c|c|c|c|c|c|}
\hline \multirow[t]{2}{*}{ Indicateur } & \multicolumn{6}{|c|}{ Hommes } & \multicolumn{6}{|c|}{ Femmes } \\
\hline & \multicolumn{2}{|c|}{$\begin{array}{c}\text { Non- } \\
\text { Autochtones }\end{array}$} & \multicolumn{2}{|c|}{$\begin{array}{l}\text { Premières } \\
\text { Nations (hors } \\
\text { réserve) }\end{array}$} & \multicolumn{2}{|c|}{ Métis } & \multicolumn{2}{|c|}{$\begin{array}{c}\text { Non- } \\
\text { Autochtones }\end{array}$} & \multicolumn{2}{|c|}{$\begin{array}{l}\text { Premières } \\
\text { Nations (hors } \\
\text { réserve) }\end{array}$} & $\%$ & Métis \\
\hline \multicolumn{13}{|l|}{ Facteurs de risque ou de protection } \\
\hline Fumeur quotidien ou occasionnel & 26,2 & $25,3-27,1$ & 44,9 & $39,1-50,7$ & 42,9 & $36,1-49,6$ & 17,8 & $17,2-18,4$ & 43,2 & $37,1-49,4$ & 35,1 & $28,7-41,5$ \\
\hline Sédentaire $^{\mathrm{a}}$ & 45,0 & $43,8-46,2$ & 51,7 & $43,0-60,4$ & 43,4 & $35,5-51,4$ & 39,6 & $38,6-40,7$ & 50,3 & $43,5-57,1$ & 40,2 & $32,5-47,9$ \\
\hline Physiquement actif & 52,4 & $51,5-53,4$ & 58,9 & $52,9-64,9$ & 53,2 & $46,7-59,7$ & 46,6 & $45,8-47,4$ & 50,1 & $44,5-55,7$ & 51,4 & $43,6-59,1$ \\
\hline $\begin{array}{l}5 \text { portions de fruits et légumes par } \\
\text { jour ou plus }\end{array}$ & 29,4 & $28,6-30,1$ & 23,5 & $18,3-28,7$ & 23,7 & $18,1-29,2$ & 42,6 & $41,8-43,4$ & 31,4 & $26,4-36,3$ & 35,8 & $28,9-42,7$ \\
\hline $\begin{array}{l}\text { Plus d'une ou plus de deux } \\
\text { consommations d'alcool par jour b }\end{array}$ & 9,8 & $9,3-10,3$ & 14,3 & $10,4-18,1$ & 16,4 & $11,5-21,3$ & 8,6 & $8,1-9,1$ & 10,6 & $7,8-13,5$ & $10,2^{\mathrm{E}}$ & $6,2-14,2$ \\
\hline $\begin{array}{l}\text { Test de Pap au cours des } 3 \text { dernières } \\
\text { années }^{\mathrm{a}}\end{array}$ & & - & & - & & - & 78,0 & $77,0-79,0$ & 76,8 & $71,7-81,8$ & 72,3 & $63,9-80,8$ \\
\hline RSOS au cours des 2 dernières années & $25,5^{\mathrm{E}}$ & $23,8-27,3$ & $28,5^{\mathrm{E}}$ & $15,7-41,3$ & 23,6 & $15,7-31,3$ & 28,2 & $26,7-29,7$ & 39,6 & $29,8-49,3$ & 22,4 & $15,2-29,6$ \\
\hline Sous-dépistage du cancer colorectal ${ }^{C}$ & 49,1 & $47,2-51,1$ & 52,3 & 39,9-64,7 & 53,4 & $40,5-66,2$ & 45,1 & $43,4-46,8$ & 38,8 & $29,0-48,5$ & 46,0 & $34,2-57,7$ \\
\hline
\end{tabular}

Source : Enquête sur la santé dans les collectivités canadiennes (données de 2007 à 2011 combinées), Statistique Canada.

Abréviations : ESCC, Enquête sur la santé dans les collectivités canadiennes; IC, intervalle de confiance; RSOS, recherche de sang occulte dans les selles.

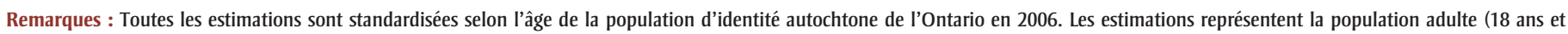

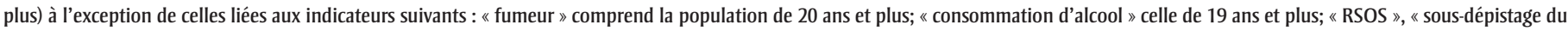
cancer colorectal » et « mammographie » celle de 50 à 74 ans; « test de Pap » celle de 21 à 69 ans.

a Indicateur non inclus dans les ESCC de 2009 et 2010. Les estimations liées à ces indicateurs représentent les données combinées des ESCC de 2007,2008 et 2011.

b Plus d'une consommation d'alcool par jour, en moyenne, chez les femmes; plus de 2 consommations d'alcool par jour, en moyenne, chez les hommes.

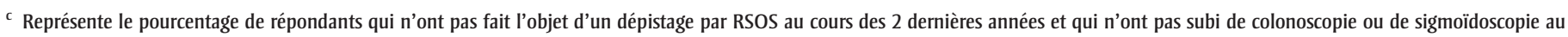
cours des 10 dernières années.

${ }^{\mathrm{E}}$ Les estimations doivent être interprétées avec prudence. Le coefficient de variation se situe entre 16,6\% et 33,29\%.

consommer au moins cinq portions de fruits et de légumes chaque jour (RC hommes $=0,72$, IC à $95 \%: 0,54$ à 0,97 ; $\mathrm{RC}$ femmes $=0,64$, IC à $95 \%: 0,51$ à 0,81 ), bien que cette différence n'était pas été significative chez les hommes après la prise en compte des différences sociodémographiques. Aucune différence significative n'a été observée entre les Métis et les non-Autochtones des deux sexes en ce qui a trait à la consommation de fruits et de légumes.

Les hommes des Premières Nations et les Métis étaient environ $50 \%$ plus nombreux que les hommes non autochtones à dépasser les limites quotidiennes recommandées de consommation d'alcool pour la prévention du cancer (RC Premières Nations $=1,50$, IC à $95 \%: 1,08$ à 2,07; RC Métis = 1,57, IC à $95 \%: 1,06$ à 2,31). Cependant, une fois les caractéristiques sociodémographiques prises en compte, l'écart observé n'était plus statistiquement significatif chez les Métis. Nous avons observé une tendance à la consommation d'alcool accrue chez les femmes des Premières Nations et les femmes métis par rapport aux femmes non autochtones, mais on a pu observer une différence statistiquement significative qu'après avoir tenu compte des caractéristiques sociodémographiques. Bien que la prise en compte de ces caractéristiques sociodémographiques ait parfois modifié la signification statistique d'un résultat, dans l'ensemble, elle n'a pas eu d'effet notable sur notre interprétation des rapports de cotes.

\section{Participation au dépistagé}

Chez les femmes, aucune différence statistiquement significative n'a été observée en ce qui concerne la participation au dépistage par mammographie ou au dépistage du cancer du col de l'utérus chez les trois groupes ethnoculturels. La plupart des femmes avaient subi un test de Pap au cours des trois dernières années (76,8 \% des femmes des Premières Nations, $72,3 \%$ des femmes métis et 78,0 \% des femmes non autochtones). En revanche, un nombre moins élevé de femmes 
FIGURE 1

Prévalence des facteurs de risque sélectionnés et participation au dépistage du cancer colorectal chez les hommes (18 ans et plus) de l'Ontario vivant hors réserve, selon le groupe d'identité autochtone, données des ESCC de 2007 à 2011 combinées

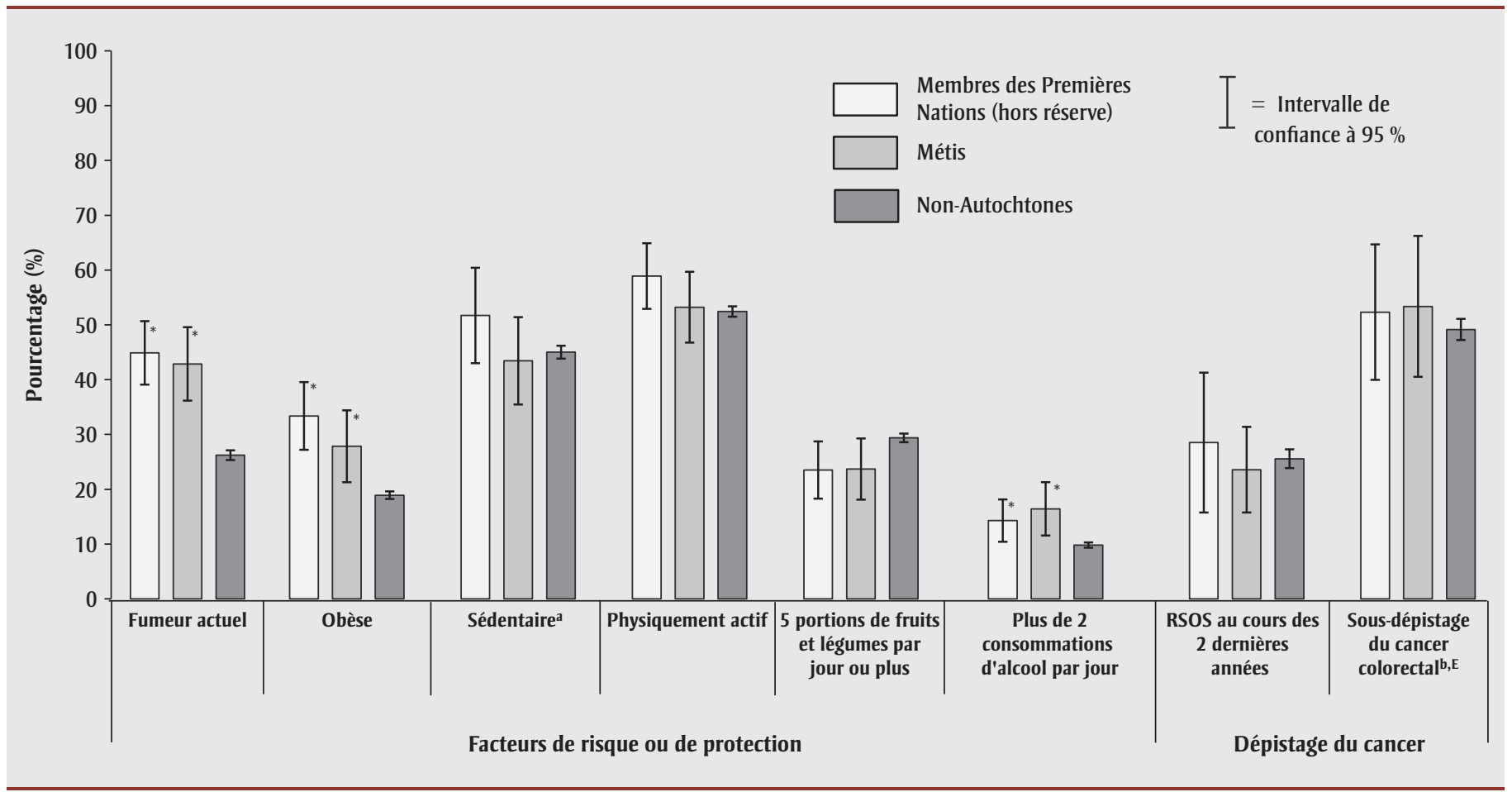

Source : Enquête sur la santé dans les collectivités canadiennes (données de 2007 à 2011 combinées), Statistique Canada.

Abréviations : ESCC, Enquête sur la santé dans les collectivités canadiennes; RSOS, recherche de sang occulte dans les selles.

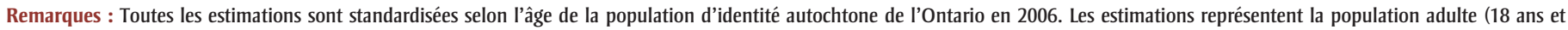

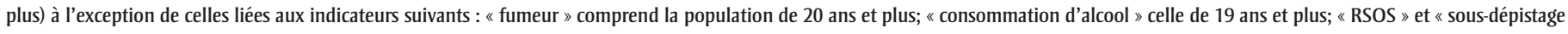
du cancer colorectal » celle de 50 à 74 ans.

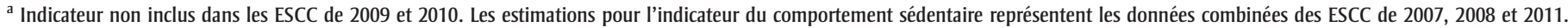

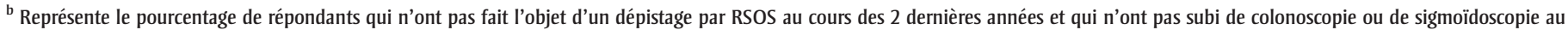
cours des 10 dernières années.

${ }^{E}$ Les estimations concernant les membres des Premières Nations et les Métis doivent être interprétées avec prudence. Le coefficient de variation se situe entre 16,6 \% et 33,29 \%.

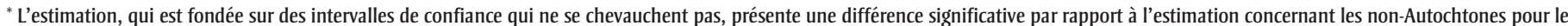
facteur de risque correspondant.

avaient déclaré avoir subi une mammographie au cours des trois dernières années, à savoir moins de $60 \%$ des femmes des Premières Nations et métis, comparativement à près de $70 \%$ des femmes non autochtones.

Pour environ la moitié des répondants, il était temps de procéder à un test de dépistage du cancer colorectal car ils n’avaient pas subi de dépistage par RSOS au cours des deux dernières années ou de coloscopie ou de sigmoïdoscopie au cours des 10 dernières années. Les taux de sousdépistage étaient plus faibles chez les femmes, et particulièrement bas (même si l'écart n'était pas statistiquement signi- ficatif) chez les femmes des Premières Nations, qui affichaient un taux de sousdépistage de 38,8 \%. Cet écart pourrait être attribuable à l'augmentation apparente de la participation au dépistage par RSOS chez les femmes des Premières Nations, qui étaient presque deux fois plus nombreuses ( $\mathrm{RC}=1,78$, IC à $95 \%$ : 1,22 à 2,59) à avoir subi ce test que leurs pairs non autochtones.

\section{Analyse}

L'analyse des résultats des ESCC 2007 à 2011 a révélé des disparités importantes entre les membres des Premières Nations, les Métis et les non-Autochtones de
l'Ontario en ce qui concerne la prévalence de certains facteurs de risque de cancer et la participation au dépistage du cancer.

Plus particulièrement, la prévalence du tabagisme et de l'obésité est apparue comme significativement plus importante chez les membres des Premières Nations et les Métis, une observation valable tant à l'échelle nationale que provinciale ${ }^{21-26}$. Nous avons observé une grande disparité en ce qui concerne les données recueillies sur les membres des réserves des Premières Nations, et les membres vivant dans les réserves présentaient des taux plus élevés de tabagisme et d'obésité que les populations vivant hors réserve ${ }^{25,26}$. 
FIGURE 2

Prévalence des facteurs de risque sélectionnés et participation au dépistage du cancer du sein (mammographie), du cancer du col de l'utérus et du cancer colorectal chez les femmes (18 ans et plus) de l'Ontario vivant hors réserve, selon le groupe d'identité autochtone, données des ESCC de 2007 à 2011 combinées

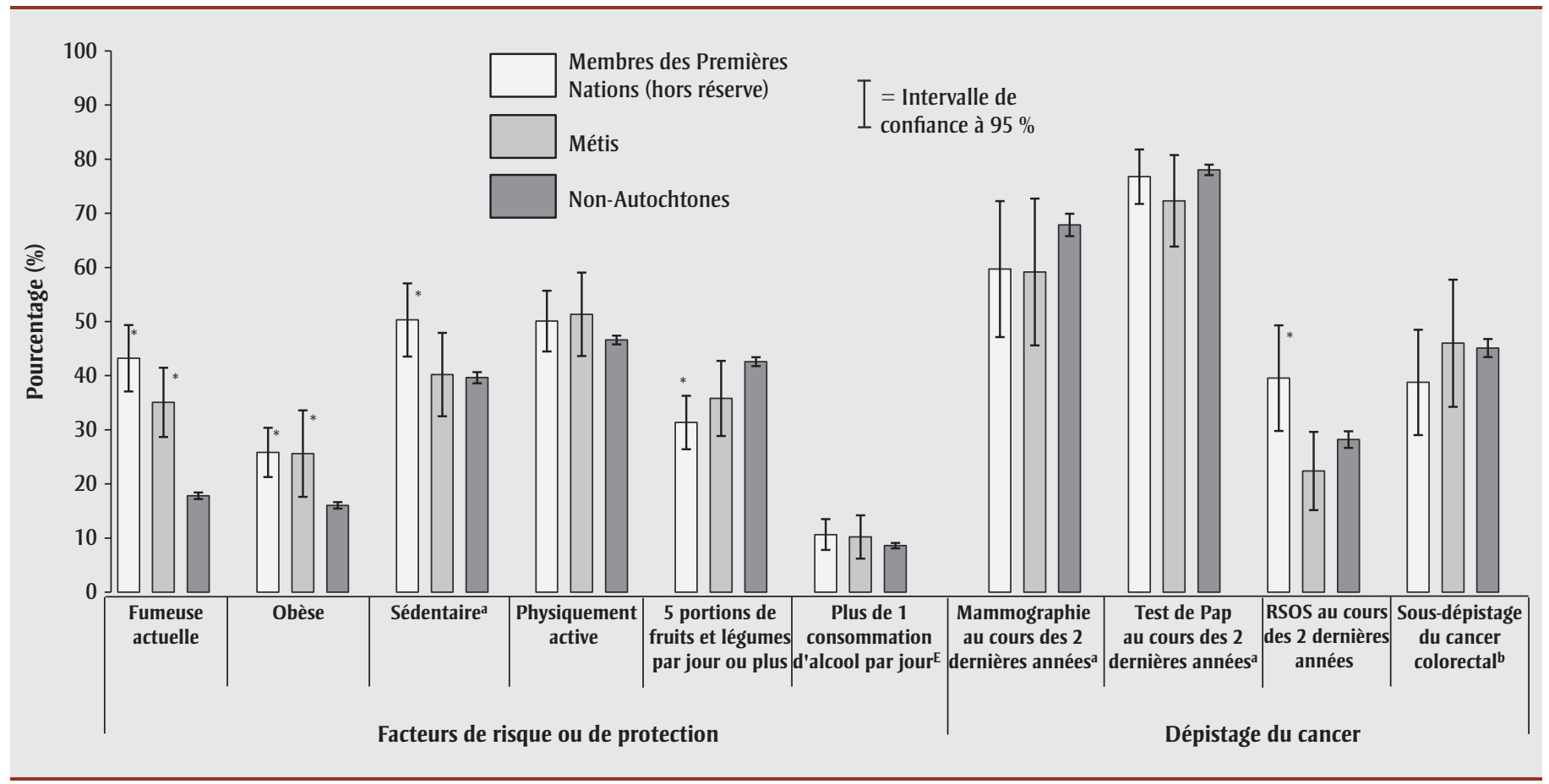

Source : Enquête sur la santé dans les collectivités canadiennes (données de 2007 à 2011 combinées), Statistique Canada.

Abréviations : ESCC, Enquête sur la santé dans les collectivités canadiennes; RSOS, recherche de sang occulte dans les selles.

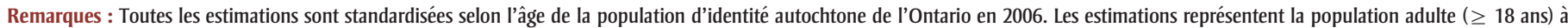

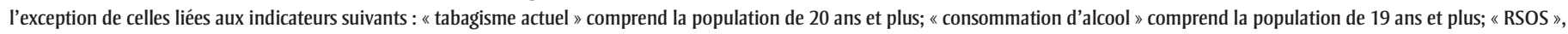
《 sous-dépistage du cancer colorectal » et « mammographie » comprennent la population de 50 à 74 ans; « test de Pap »comprend la population âgée de 21 à 69 ans.

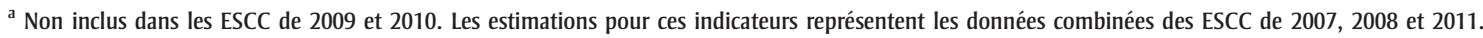

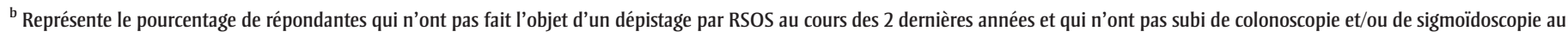
cours des 10 dernières années.

${ }^{\text {E }}$ Les estimations concernant les membres des Premières Nations et les Métis doivent être interprétées avec prudence. Le coefficient de variation se situe entre 16,6 \% et 33,29 \%.

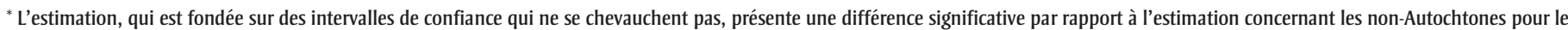
facteur de risque correspondant.

Bien que les données aient semblé indiquer une diminution entre 2001 et $2008^{27}$ du taux de tabagisme et d'obésité chez les populations autochtones vivant hors réserve, la prévalence de ces deux facteurs de risque demeure nettement supérieure à celle observée au sein de la population générale. Les cancers colorectal et du poumon ${ }^{27-29}$ sont en grande partie responsables de l'accroissement rapide de l'incidence du cancer chez la population autochtone. Or ces deux types de cancers ont été associés au tabagisme et le cancer colorectal a été associé à l'obésité $^{7,8,30}$. Il est donc impératif d'accorder la priorité aux interventions visant à réduire ces facteurs de risque négatifs chez la population autochtone.

Les analyses ont également démontré que la consommation de fruits et de légumes était moins élevée et la consommation d'alcool plus importante chez les membres des Premières Nations que dans la population non autochtone. Il ressort de données antérieures que, en plus de consommer peu de fruits et de légumes, les femmes des Premières Nations vivant hors réserve présentent un apport calorique quotidien moyen supérieur, que les femmes autochtones consomment moins de portions de produits céréaliers et que les hommes autochtones consomment moins de portions de produits laitiers que la population générale $^{31}$. Cette situation est préoccupante, car plusieurs études ont établi un lien entre une mauvaise alimentation et un risque accru de cancer ${ }^{10}$.

À notre connaissance, aucune étude sur la population autochtone au Canada n'a utilisé d'indicateur de consommation d'alcool basé sur les recommandations pour la prévention du cancer similaire à celui que nous avons employé, même si une augmentation de la fréquence de la consommation occasionnelle excessive d'alcool a été signalée chez les membres 


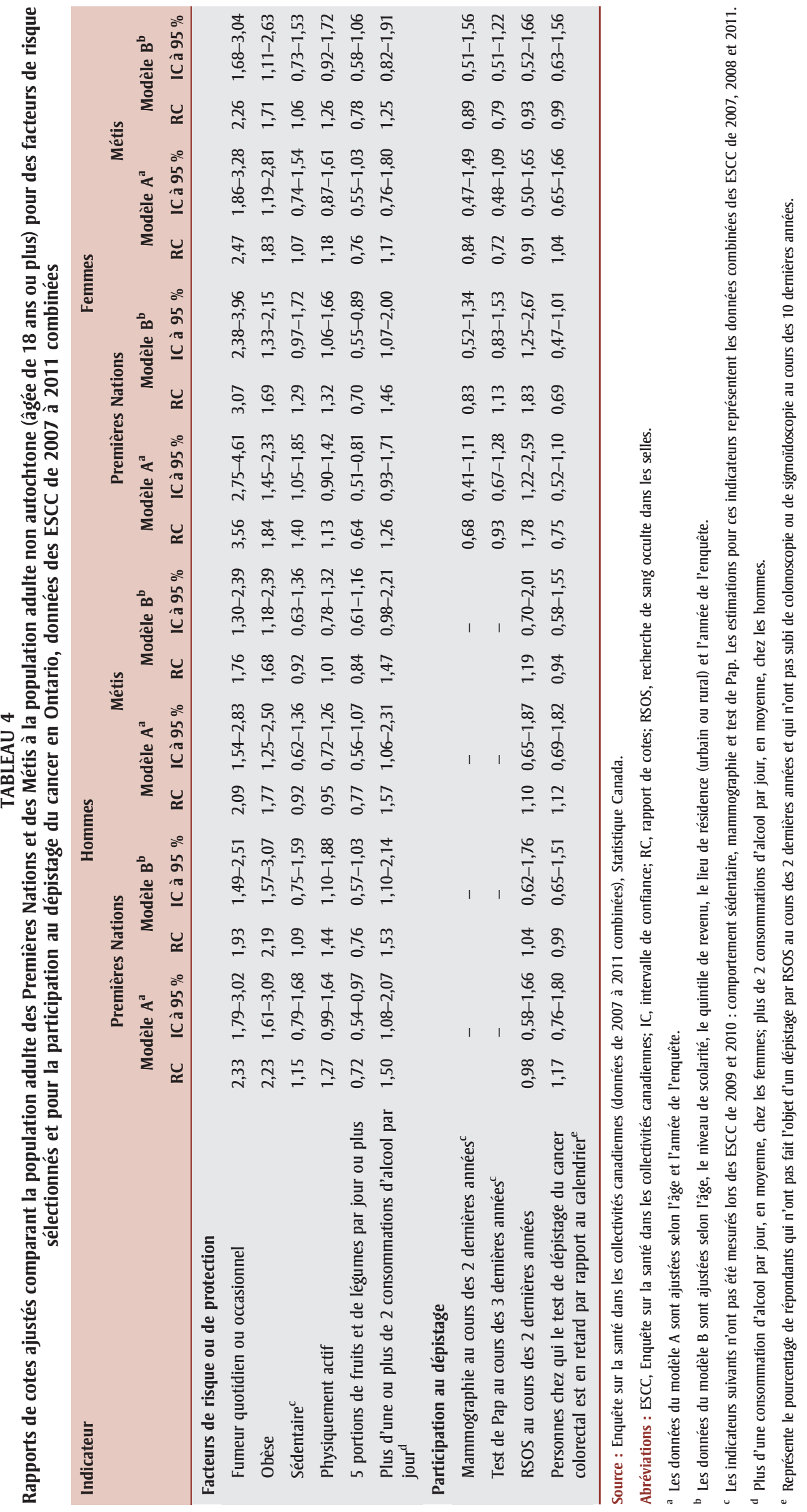


des Premières Nations et les Métis de l'Ontario $^{5,23}$. Compte tenu des données antérieures révélant une plus faible prévalence de la consommation abusive d'alcool dans la population autochtone par rapport à la population générale ${ }^{22}$, nos résultats ainsi que d'autres données plus récentes $^{6,24}$ laissent entrevoir une évolution future à la hausse de cette consommation à risque au sein de la population autochtone.

Aucune différence significative n'a été observée entre les trois groupes ethnoculturels en ce qui concerne la participation au dépistage du cancer du sein et du cancer du col de l'utérus. Nous avons observé une tendance à la baisse pour ce qui est des taux de mammographie chez les femmes des Premières Nations et les femmes métis, mais, fait important, aucun des groupes ethnoculturels n'a atteint l'objectif national d'un taux de couverture de $70 \%{ }^{32}$. Nous disposons de peu de données sur la participation au dépistage par mammographie chez les Autochtones, mais une étude menée au Manitoba a révélé une participation nettement plus faible chez les femmes des Premières Nations vivant dans une réserve que chez les femmes vivant en milieu rural ${ }^{33}$. De plus, il ressort d'une étude antérieure que les femmes des minorités visibles au Canada sont moins nombreuses que les femmes blanches à déclarer avoir subi une mammographie ${ }^{34}$.

Notre étude révèle que $70 \%$ des femmes ont déclaré avoir subi un test de Pap au cours des trois années précédentes, ce qui confirme des résultats de recherche antérieurs ${ }^{34}$. Cependant, malgré une participation adéquate au dépistage, le fardeau du cancer du col de l'utérus chez les Autochtones est disproportionnellement élevé, ce qui semble indiquer que des mesures préventives supplémentaires ou différentes seraient nécessaires ${ }^{35}$.

Comparativement aux femmes non autochtones, les femmes des Premières Nations étaient beaucoup plus nombreuses à déclarer avoir fait l'objet d'un dépistage par RSOS au cours des deux dernières années, et le taux de sousdépistage du cancer colorectal était inférieur chez celles-ci si l'on prenait en compte les données sur la colonoscopie et la sigmoïdoscopie. Si l'on interprète conjointement ces deux indicateurs de dépistage du cancer colorectal, cela laisse entrevoir une plus forte propension à la participation au dépistage par RSOS (par opposition à la colonoscopie ou à la sigmoïdoscopie) chez les membres des Premières Nations, comparativement aux Métis et aux non-Autochtones. Ce taux de participation pourrait découler, du moins en partie, de la mise en œuvre en 20082009 d'une initiative éducative d'Action Cancer Ontario visant à sensibiliser les communautés des Premières Nations à l'égard du cancer colorectal, de la prévention et du dépistage par RSOS ${ }^{36}$.

Il est particulièrement important de tenir compte des facteurs sociaux dans les études sur la santé des Autochtones. Comparativement à la population non autochtone, la population autochtone au Canada risque davantage de vivre dans la pauvreté, de déclarer un revenu familial plus faible et de présenter un niveau de scolarité inférieur ${ }^{21,23,37}$. En outre, les membres des Premières Nations et les Métis sont plus nombreux à vivre en milieu rural, et le fait d'habiter en milieu rural a été associé à un accès plus difficile aux services de santé ainsi qu'à une augmentation du risque de tabagisme, de surpoids et de mauvaise autoévaluation de la santé ${ }^{38}$. Nous avons donc étudié l'impact du revenu, de l'éducation et du lieu de résidence (milieu rural ou urbain) mais, après la prise en compte de ces facteurs, nous avons observé très peu de changement entre la population autochtone et la population non autochtone dans la probabilité de présenter des facteurs de risque et dans la participation au dépistage. Cette constatation semble confirmer la robustesse de l'origine ethnique comme déterminant des facteurs de risque pour la santé liés au mode de vie chez les membres des Premières Nations et les Métis.

\section{Forces et limites}

L'origine ethnique, le statut socioéconomique et le lieu de résidence sont des déterminants connus des facteurs de risque de maladie chronique les plus courants. Nous n'avons pas été en mesure de tenir compte d'autres déterminants pourtant importants de la santé et de la maladie dans notre étude. Par exemple, des facteurs plus éloignés comme le système de soins de santé, le racisme ou le capital social ont des effets indirects importants sur la santé des Autochtones, mais ces facteurs ne peuvent pas être pris en compte à l'aide des données de l'ESCC ${ }^{23,39}$. De plus, nous avons été en mesure d'examiner la prévalence de chacun des facteurs de risque pris individuellement mais nous n'avons pas évalué les relations entre ceux-ci. Par exemple, nous avons estimé la prévalence de l'activité physique, de l'alimentation et de l'obésité mais, bien que nous sachions que l'activité physique et l'alimentation constituent des déterminants importants de l'obésité $^{40}$, nous n'avons pas étudié leur relation dans cette étude. Cette lacune pourrait inciter des chercheurs à mener des études de modélisation causale axées sur les facteurs de risque multiples et les maladies chroniques au sein de la population autochtone et non autochtone.

Le fait que les personnes vivant dans des réserves aient été exclues des ESCC constitue une autre limite à nos travaux. Les données probantes de l'Enquête régionale sur la santé des Premières Nations, qui porte sur les membres des Premières Nations vivant dans les réserves, semblent indiquer qu'outre une prévalence plus élevée du tabagisme et de l'obésité chez les membres des communautés vivant dans les réserves, le taux d'activité physique semble être plus faible et les taux de dépistage du cancer du col de l'utérus et du cancer du sein similaires à ceux des résultats de notre étude ${ }^{26}$.

En regroupant les réponses obtenues dans le cadre de cinq ESCC, nous avons été en mesure de faire des estimations de prévalence pour les membres des Premières Nations et les Métis de l'Ontario, ce qui constitue l'une des forces de nos travaux. Cependant, les estimations réalisées au moyen de cette approche ont comme inconvénient d'offrir une prévalence moyenne pour une période plutôt qu'un état de santé actualisé des populations étudiées.

Enfin, comme les données de l'enquête ont été recueillies par autodéclaration, nos résultats peuvent présenter un biais de 
désirabilité sociale, qui conduit à une sous-déclaration des comportements considérés comme non souhaitables sur le plan social et à une surdéclaration des comportements considérés comme désirables. On peut cependant penser que cet effet est similaire d'un groupe culturel à l'autre et n'aurait donc pas d'effet significatif sur la prévalence relative d'un facteur de risque.

Les analyses présentées dans cette étude montrent, malgré ces limites, que l'ESCC peut fournir des estimations des facteurs de risque pour les membres des Premières Nations vivant hors réserve, les Métis et la population non autochtone de l'Ontario au moyen d'indicateurs uniformes entre ces populations. En outre, notre étude fournit de nouvelles données probantes sur l'état de santé des membres des Premières Nations et des Métis de l'Ontario, notamment en ce qui concerne le risque de cancer, par le biais de l'évaluation des facteurs de risque par rapport aux lignes directrices en matière de prévention du cancer, par exemple touchant les comportements sédentaires, la participation au dépistage du cancer et la mesure de la consommation d'alcool.

\section{Conclusion}

L'estimation de la prévalence des facteurs de risque et de la participation au dépistage du cancer chez les membres des Premières Nations et les Métis est essentielle à la planification et à la prestation des services de prévention primaire et secondaire auprès de cette population. De telles analyses devraient être répétées au fil du temps pour surveiller les tendances et définir les cibles d'intervention. Les données sur l'augmentation de la prévalence des facteurs de risque de maladie chronique chez les membres des Premières Nations et les Métis présentées dans cette étude militent en faveur des recommandations provinciales selon lesquelles des mesures adéquates et adaptées sur le plan culturel doivent être prises pour agir sur ces facteurs en vue de réduire le fardeau du cancer en particulier et des maladies chroniques en général pour la génération actuelle et celles qui suivront ${ }^{41}$.

\section{Références}

1. Tjepkema $M$, Wilkins $R$, Senécal $S$, Guimond E, Penney C. La mortalité chez les Métis et les Indiens inscrits adultes au Canada : étude de suivi sur 11 ans. Rapports sur la santé. 2009. (Statistique Canada, n॰82-003-X au catalogue)

2. Conseil canadien de la santé. L'état de santé des Premières nations, des Métis et des Inuits du Canada. Toronto (Ont.) : Conseil canadien de la santé; 2005. PDF (664 Ko) téléchargeable à partir du lien : http:// publications.gc.ca/collections/collection _2012/ccs-hcc/H174-37-2005-1-fra.pdf

3. Société canadienne du cancer. Statistiques canadiennes sur le cancer 2013. Ottawa (Ont.) : Société canadienne du cancer; 2013. Consultable en ligne à partir de la page : http://www.cancer.ca/fr-ca/cancer -information/cancer-101/canadian-cancer -statistics-publication/?region $=$ on

4. Waldram JB, Herring DA, Young TK. Aboriginal health in Canada: historical, cultural, and epidemiological perspectives. $2^{\mathrm{e}}$ éd. Toronto (Ont.) : University of Toronto Press; 2006.

5. Statistique Canada. Enquête nationale auprès des ménages, 2011 : Les peuples autochtones au Canada : Premières Nations, Métis et Inuits. Ottawa (Ont.) : Statistique Canada; 2013. (Statistique Canada, n॰99-011-X2011001 au catalogue). Consultable en ligne à la page : http:// www12.statcan.gc.ca/nhs-enm/2011/as-sa /99-011-x/99-011-x2011001-fra.cfm

6. Gionet L, Roshanafshar S. Certains indicateurs de la santé des membres des Premières nations vivant hors réserve, des Métis et des Inuits. Coup d'œil sur la santé. 2013;(janv):1-15. (Statistique Canada, $n^{\circ}$ 82-624-X au catalogue)

7. National Cancer Institute (NCI). Tobacco facts [Internet]. Bethesda (MD) : National Cancer Institute; 2013 [Consulté le 13 juillet 2013]. Consultable en ligne à la page : http://www .cancer.gov/cancertopics/tobacco/smoking

8. National Cancer Institute (NCI). Obesity and cancer risk [Internet]. Bethesda (MD) : National Institutes of Health; 2012 [mis à jour le 3 janvier 2012; consulté le 13 juillet 2013]. Consultable en ligne à la page : http:// www.cancer.gov/cancertopics/factsheet/Risk /obesity
9. National Cancer Institute (NCI). Physical activity and cancer [Internet]. Bethesda (MD) : National Cancer Institute; 2009 [mis à jour le 22 juillet 2009; consulté le 13 juillet 2013]. Consultable en ligne à la page : http://www.cancer.gov/cancertopics /factsheet/prevention/physicalactivity

10. Cancer Research UK. Diet and cancer: the evidence [Internet]. London (UK) : Cancer Research UK; 2009 [consulté le 13 juillet 2013]. Consultable en ligne à la page : http:// www.cancerresearchuk.org/cancer-info /healthyliving/dietandhealthyeating/how doweknow/diet-and-cancer-the-evidence

11. National Cancer Institute (NCI). Alcohol and cancer risk [Internet]. Bethesda (MD) : National Cancer Institute; 2013 [mis à jour le 24 juin 2013; consulté le 13 juillet 2013]. Consultable en ligne à la page : http://www .cancer.gov/cancertopics/factsheet/Risk /alcohol

12. Lynch BM. Sedentary behavior and cancer: a systematic review of the literature and proposed biological mechanisms. Cancer Epidemiol Biomarkers Prev. 2010;19(11): 2691-2709.

13. Statistique Canada. Autres périodes de référence - Enquête sur la santé dans les collectivités canadiennes - Composante annuelle [Internet]. Ottawa (Ont.) : Statistique Canada [consultéle 3 novembre 2013]. Consultable en ligne à la page : http://www23.statcan.gc.ca /imdb/p2SV_f.pl?Function = getInstanceList\& SurvId $=3226 \&$ SurvVer $=2 \&$ InstaId $=15282 \&$ SDDS $=3226 \&$ lang $=$ en $\& d b=i m d b \& a d m=$ $8 \&$ dis $=2$

14. Thomas S, Wannell B. Combiner les cycles de l'Enquête sur la santé dans les collectivités canadiennes. Rapports sur la santé. 2009;20(1):59-65.

15. Action Cancer Ontario. Les mammographies [Internet]. Toronto (Ont.) : Action Cancer Ontario [mis à jour le 18 octobre 2012; consulté le 16 juillet 2013]. Consultable en ligne à la page : https://fr.cancercare.on.ca/pcs /screening/breastscreening/mammograms/ 
16. Action Cancer Ontario. Lignes directrices ontariennes sur la cytologie de dépistage du cancer du col [Internet]. Toronto (Ont.) : Action Cancer Ontario; [mis à jour le 24 avril 2013; consulté le 16 juillet 2013]. Consultable en ligne à la page : https://fr.cancercare.on .ca/pcs/screening/cervscreening/screening guidelines/

17. Action Cancer Ontario. À propos du dépistage du cancer colorectal [Internet]. Toronto (Ont.) : Action Cancer Ontario; [mis à jour le 3 mai 2011; consulté le 16 juillet 2013]. Consultable en ligne à la page : https://fr.cancercare.on.ca/pcs/screening /coloscreening/aboutcolscreening/

18. Association of Public Health Epidemiologists in Ontario (APHEO). 5A Smoking Status [Internet]. Toronto (Ont.) : Association of Public Health Epidemiologists in Ontario; 2009 [mis à jour le 29 juin 2009; cité le 9 juillet 2013]. Consultable en ligne à la page : http://www.apheo.ca/index.php?pid = 117

19. World Cancer Research Fund/American Institute for Cancer Research. Food, nutrition, physical activity, and the prevention of cancer: a global perspective. Washington (DC) : World Cancer Research Fund/ American Institute for Cancer Research; 2007.

20. Statistique Canada. Enquête sur la santé dans les collectivités canadiennes (ESSC). Composante annuelle - Questionnaire de 2011. Ottawa (Ont.) : Statistique Canada; 2011. PDF (4 Mo) téléchargeable à partir du lien : http://www23.statcan.gc.ca/imdb-bmdi /instrument/3226_Q1_V8-fra.pdf

21. Garner R, Carrière G, Sanmartin $C$ et l'équipe de recherche de l'Initiative sur les données longitudinales administratives et sur la santé. La santé des adultes chez les Premières Nations vivant hors réserve, les Inuits, et les Métis au Canada : l'incidence du statut socioéconomique sur les inégalités en matière de santé. Série de documents de travail de la recherche sur la santé. Juin 2010. (Statistique Canada, n 82 622-X au catalogue)

22. Lix LM, Bruce S, Sarkar J, Young TK. Facteurs de risque et problèmes de santé chroniques chez les Autochtones et les nonAutochtones . Rapports sur la santé. 2009; 20(4):23-32. (Statistique Canada, n॰82-003XPF au catalogue)
23. Reading CL, Wien F. Inégalités en matière de santé et déterminants sociaux de la santé des peuples autochtones [Internet]. Prince George (C.-B.) : Centre de collaboration nationale de la santé autochtone; 2009 [consulté le 16 juillet 2013]. PDF (1,3 Mo) téléchargeable à partir du lien : http:// www.nccah-ccnsa.ca/Publications/Lists /Publications/Attachments/46/health_ inequalities_FR_web.pdf

24. Withrow D, Kewayosh A, Marrett L. Cancer in the Métis nation of Ontario: clinical significance report [Internet]. Ottawa (Ont.) : Métis Nation of Ontario; 2012. PDF (433 Ko) téléchargeable à partir du lien : http:// www.metisnation.org/media/229177/mno $\% 20$ cancer $\% 20$ clinical $\% 20$ significance $\% 20$ report $\% 20 \% 2829$-mar-2012\%29.pdf

25. L'Enquête régionale sur la santé des Premières Nations (ERS) (2002-2003). Résultats de l'enquête sur les enfants, les jeunes et les adultes des Premières Nations. Ottawa (Ont.) : Centre de gouvernance de l’information des Premières Nations; 2005.

26. L’Enquête régionale sur la santé des Premières Nations (ERS) Phase 2 (2008/ 2010) Rapport national sur les adultes, les adolescents et les enfants qui vivent dans les communautés des Premières Nations. Ottawa (Ont.) : Centre de gouvernance de l'information des Premières Nations; 2012.

27. Action Cancer Ontario. Stratégie pour la lutte contre le cancer chez les peuples autochtones II [Internet]. Toronto (Ont.) : Action Cancer Ontario; [sans date]. PDF (5,74 Mo) téléchargeable à partir du lien : https://fr.cancercare.on.ca/common/pages /UserFile.aspx?fileId $=257017$

28. Marrett LD, Chaudhry M. Cancer incidence and mortality in Ontario First Nations, 1968-1991 (Canada). Cancer Causes Control. 2003;14:259-68.

29. Kelly J, Lanier A, Santos M et collab. Cancer among the circumpolar Inuit, 1989-2003. II. Patterns and trends. Int J Circumpolar Health. 2008;67(5):408-20.

30. Zhao J, Halfyard B, Roebothan B et collab. Tobacco smoking and colorectal cancer: a population-based case-control study in Newfoundland and Labrador. Can J Public Health. 2010;101(4):281-9.
31. Statistique Canada. Enquête sur la santé dans les collectivités canadiennes : vue d'ensemble des habitudes alimentaires des Canadiens. [Internet]. Ottawa (Ont.) : Le Quotidien; 6 juil. 2006 [consulté le 16 juillet 2013]. Consultable en ligne à la page : http://www.statcan.gc.ca/daily-quotidien /060706/dq060706b-fra.htm

32. Action Cancer Ontario. Dépistage du cancer du sein [Internet]. Toronto (Ont.) : Action Cancer Ontario; 28 nov. 2012 [consulté le 16 juillet 2013]. Consultable en ligne à la page : https://fr.cancercare.on.ca/pcs/screening /breastscreening/?WT.mc_id = /obsp

33. Elias B, Kliewer EV, Hall M, et al. The burden of cancer risk in Canada's indigenous population: a comparative study of known risks in a Canadian region. Int J Gen Med. 2011;4:699-709.

34. Quan H, Fong A, De Coster C, et al. Variation in health services utilization among ethnic populations. CMAJ. 2006;174(6):787-91.

35. Demers AA, Kliewer EV, Remes O, et al. Cervical cancer among Aboriginal women in Canada. CMAJ. 2012;184(7):743-44.

36. Sattler P. "Let's Take a Stand Against...Colorectal Cancer!” Evaluation report [Internet]. Toronto (Ont.) : Cancer Care Ontario; 2011 May [consulté le 16 juillet 2013]. Consultable en ligne à la page : https://www.cancercare.on.ca/cms/one .aspx? objectId $=37272 \&$ contextId $=1377$

37. Macaulay AC. Improving aboriginal health: how can health care professionals contribute? Can Fam Physician. 2009; 55(4):334-6.

38. Mitura V, Bollman RD. La santé des Canadiens des régions rurales : une comparaison rurale-urbaine des indicateurs de santé [Internet]. Bulletin d'analyse Régions rurales et petites villes du Canada. 2003;4(6):1-23. (Statistique Canada, n²1006-XIF au catalogue). PDF (185 Ko) téléchargeable à partir du lien : http://www .statcan.gc.ca/pub/21-006-x/21-006-x2002006 -fra.pdf

39. King M, Smith A, Gracey M. Indigenous health part 2: the underlying causes of the health gap. Lancet. 2009;374(9683):76-85. 
40. National Heart, Blood and Lung Institute (NHLBI). What causes overweight and obesity? [Internet]. Bethesda (MD) : National Heart, Blood and Lung Institute; 2013 [consulté le 16 juillet 2013]. Consultable en ligne à la page : http://www.nhlbi.nih.gov/health /health-topics/topics/obe/causes.html

41. Manson H, Rabeneck L, Allison KR et collab. Agir pour prévenir les maladies chroniques : recommandations pour améliorer la santé de la population ontarienne. Toronto (Ont.) : Agence ontarienne de protection et de promotion de la santé; 2012. 